\title{
Specific Heat and Magnetism of $\mathrm{LuFe}_{6} \mathrm{Al}_{6}$
}

\section{P. SVOBODA}

Charles University, Faculty of Mathematics and Physics

Department of Condensed Matter Physics

Ke Karlovu 5, 12116 Prague 2, Czech Republic

A.V. Andreev, E. Šantavá And J. ŠEBeK

Institute of Physics ASCR, Na Slovance 2, 18221 Prague 8, Czech Republic

Here we present the results of the zero-field specific heat study of the $\mathrm{LuFe}_{6} \mathrm{Al}_{6}$ single crystal. The specific heat data were analyzed as a sum of the phonon, electronic, and magnetic contributions, respectively. The analysis of the phonon part involves three acoustic and 36 optical branches, respectively, all of them corrected for the anharmonicity. The magnetic part of the specific heat was obtained by subtracting the electronic and the phonon part from the experimental specific heat and the magnetic entropy was calculated.

PACS numbers: 75.40.Cx, 65.40.Ba

\section{Introduction}

Intermetallic compound $\mathrm{LuFe}_{6} \mathrm{Al}_{6}$ belongs to the $\mathrm{REFe}_{12-x} \mathrm{X}_{x}$ series $(\mathrm{RE}=$ rare earth, $\mathrm{X}=p$-metal) and crystallizes in the tetragonal crystal structure of the $\mathrm{ThMn}_{12}$ type. As binary $\mathrm{REFe}_{12}$ compounds do not exist, the partial substitution of iron by the $\mathrm{X}$ element is necessary to stabilize the $\mathrm{ThMn}_{12}$-type structure. The compounds of this type of structure and of high iron content have been considered as good candidates for hard magnetic materials (e.g. [1, 2]) and extensively studied in recent years. At lower content of iron, these compounds may display complex magnetic orderings and structures due to competing $\mathrm{RE}-\mathrm{Fe}$ and $\mathrm{Fe}-\mathrm{Fe}$ magnetic couplings.

\section{Experimental}

The single crystal of $\mathrm{LuFe}_{6} \mathrm{Al}_{6}$ has been grown by the Czochralski pulling method. The X-ray diffraction has confirmed the $\mathrm{ThMn}_{12}$-type structure with lattice parameters $a=859.3 \mathrm{pm}, c=502.1 \mathrm{pm}$. 


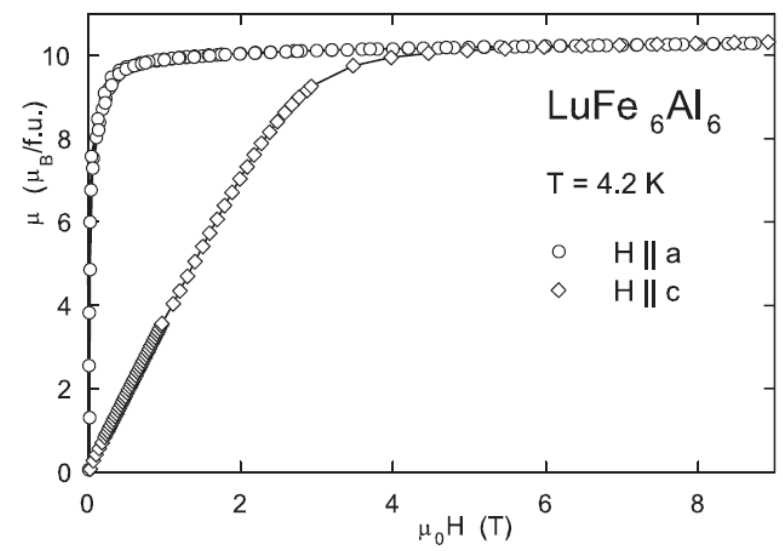

Fig. 1. Magnetization curves of $\mathrm{LuFe}_{6} \mathrm{Al}_{6}$ along principal crystallographic axes.

The magnetization measurements of this single crystal revealed that $\mathrm{LuFe}_{6} \mathrm{Al}_{6}$ orders ferromagnetically below the Curie temperature $T_{\mathrm{C}}=325 \mathrm{~K}$ with spontaneous magnetic moment $\mu=10 \mu_{\mathrm{B}}$ per formula unit at $T=4.2 \mathrm{~K}$. The magnetization curves in Fig. 1 clearly indicate the easy-plane magnetic anisotropy in this compound. Further details of the crystal growth and magnetic properties will be published elsewhere.

In this paper we concentrate on the specific heat of $\mathrm{LuFe}_{6} \mathrm{Al}_{6}$. About $10 \mathrm{mg}$ of the crystal was used for the specific heat $C(T)$ study using the commercial PPMS apparatus (Quantum Design) in the temperature range $2-370 \mathrm{~K}$, the measurement was performed using relaxation method.

\section{Results and discussion}

The zero-field specific heat has been analyzed as a sum of the phonon, electronic, and magnetic contributions, respectively. The electronic specific heat $C_{\mathrm{e}}$ was described by simple Sommerfeld term $C_{\mathrm{e}}=\gamma T$, the $C / T$ vs. $T^{2}$ plot yielded the $\gamma$-coefficient about $\gamma=68 \mathrm{~mJ} /\left(\mathrm{mol} \mathrm{K}^{2}\right)$.

The analysis is based on the possible realistic description of the phonon specific heat $C_{\mathrm{ph}}$. In $\mathrm{LuFe}_{6} \mathrm{Al}_{6}$ the phonon spectrum consists of 3 acoustic branches and 36 optical ones. The specific heat of all phonon branches is corrected for the anharmonicity, which also accounts for the discrepancy between the isobaric and the isochoric specific heat (e.g. [3]).

3 acoustic branches are described by the Debye term $C_{\mathrm{D}}$ and for the 36 optical ones the Einstein model $C_{\mathrm{E}}$ can be used in the form (e.g. [4]):

$$
C_{\mathrm{ph}}=R\left(\frac{9 C_{\mathrm{D}}}{1-\alpha_{\mathrm{D}} T}+\sum_{i=1}^{36} \frac{C_{\mathrm{E} i}}{1-\alpha_{\mathrm{E} i} T}\right)
$$

where $R$ is a gas constant. The analysis yields the characteristic temperatures $\theta_{\mathrm{D}}$ and $\theta_{\mathrm{E}}$ and corresponding anharmonicity coefficients $\alpha_{\mathrm{D}}$ and $\alpha_{\mathrm{E}}$, respectively. 


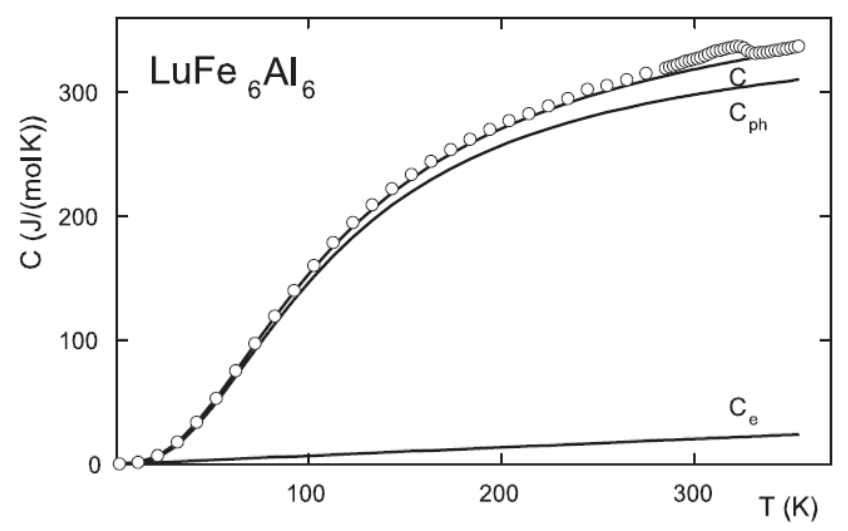

Fig. 2. Specific heat of $\mathrm{LuFe}_{6} \mathrm{Al}_{6}$ in comparison with the fit of total, phonon, and electronic specific heat, respectively.

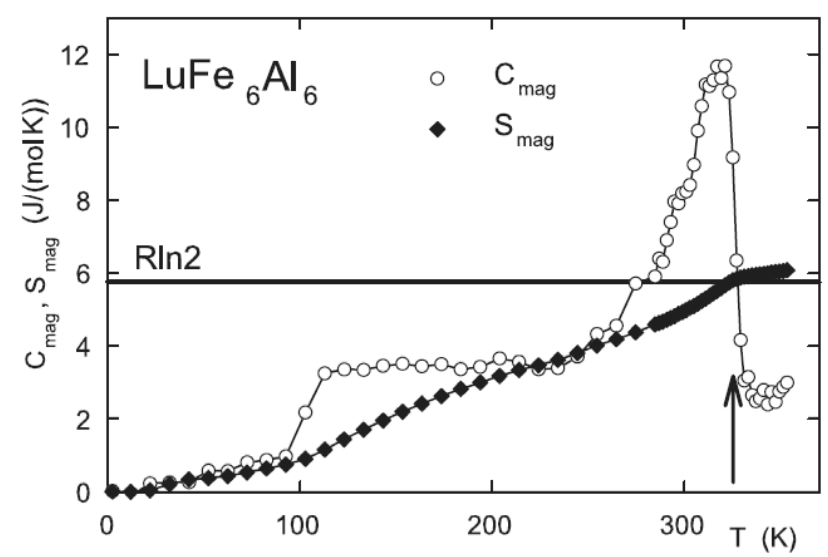

Fig. 3. Magnetic specific heat $C_{\mathrm{mag}}$ and magnetic entropy $S_{\mathrm{mag}}$ of $\mathrm{LuFe}_{6} \mathrm{Al}_{6}$. The arrow indicates $T_{\mathrm{C}}$.

Trying to reduce the number of adjustable parameters as much as possible and considering the experimental error, several optical branches were grouped using the same Einstein temperature.

As the non-magnetic analogue is not available, the agreement in the low temperature (below $100 \mathrm{~K}$ ) and above the Curie temperature, where the magnetic contribution is negligible, was used as a criterion of the goodness of the fit. It has to be noted that using just the simple Debye approach we were not able to get good agreement of the fit and physically realistic value of the magnetic entropy. The best grouping scheme with the best fit values are summarized in Table, the agreement with the experimental data is shown in Fig. 2.

The nonmagnetic part $C_{\mathrm{ph}}+C_{\mathrm{e}}$ was subtracted from the $C(T)$ data and the magnetic entropy $S_{\text {mag }}$ was calculated from the resulting magnetic specific heat $C_{\text {mag }}$ using numerical integration of $C_{\mathrm{mag}} / T$. 


\section{TABLE}

Results of the phonon specific heat analysis, $n$ denotes the degeneracy of Debye (D) and Einstein (E) branches, respectively.

\begin{tabular}{c|c|c|c}
\hline \hline Branch & $n$ & $\theta[\mathrm{K}]$ & $\alpha\left[10^{-4} \mathrm{~K}^{-1}\right]$ \\
\hline D & 3 & $163 \pm 2$ & $2.0 \pm 0.5$ \\
E1 & 8 & $198 \pm 2$ & $1.5 \pm 0.3$ \\
E2 & 10 & $323 \pm 5$ & $1.0 \pm 0.2$ \\
E3 & 12 & $390 \pm 5$ & $1.0 \pm 0.3$ \\
E4 & 6 & $596 \pm 8$ & $1.0 \pm 0.5$
\end{tabular}

As can be seen from Fig. 3 the values of $S_{\text {mag }}$ just above the ordering temperature are close to $R \ln 2$, as can be expected for the ferromagnetic and strongly anisotropic system. The step in the $C_{\text {mag }}$ data above $100 \mathrm{~K}$ has to be investigated in more detail in the future.

\section{Acknowledgments}

This work is a part of the research plan MSM 0021620834, financed by the Ministry of Education of CR, also supported by GACR grant 106/05/0393.

\section{References}

[1] H.S. Li, J.M.D. Coey, in: Handbook of Magnetic Materials, Vol. 6, Ed. K.H.J. Buschow, North-Holland, Amsterdam 1991, p. 1.

[2] K.H.J. Buschow, in: Supermagnets, Hard Magnetic Materials, Eds. G.J. Long, F. Grandjean, Kluwer, Dordrecht 1991, p. 49.

[3] C.A. Martin, J. Phys., Condens. Matter 3, 5967 (1991).

[4] P. Svoboda, P. Javorský, M. Diviš, V. Sechovský, F. Honda, G. Oomi, A.A. Menovsky, Phys. Rev. B 63, 212408 (2001). 